\title{
Postpartale Psychosen
}

\author{
Michael Grube \\ Klinik für Psychiatrie und Psychotherapie an den Städtischen Kliniken Frankfurt-Höchst, \\ Lehrkrankenhaus der Universität Frankfurt
}

psychoneuro 2005; 31 (9): 441-444

S chon im Corpus Hippocraticum, einer Sammlung der in der Zeit vom 6. Jh. v. Chr. bis 1. Jh. n. Chr. von verschiedenen Autoren verfassten 63 Schriften der griechischen Medizin, fanden die psychiatrischen Störungen nach Niederkunft und im Wochenbett Erwähnung. Der portugiesische Mediziner Esteban Rodrigo de Castro beschrieb bereits 1617 anhand von Falldarstellungen psychische Störungen im Wochenbett in seiner Schrift: „De melancholica, mania, delirio, et epilepsia puerperarum“ (18). Im Jahr 1858 widmete Louis Victor Marcé eine Monografie den psychiatrischen Störungen in Schwangerschaft, Wochenbett und Stillzeit (24). Auch in deutschsprachigen Ländern steigt inzwischen das Interesse an postpartalen psychiatrischen Störungen, insbesondere gefördert durch zwei Fachgesellschaften (Marcé-Gesellschaft für perinatale Psychiatrie e.V., Gesellschaft für die psychische Gesundheit von Frauen). Synonym werden die Begriffe „Wochenbettpsychose“, „Kindbettpsychose“ und „Puerperalpsychose“ gebraucht (29). Sie beziehen sich auf das Auftreten einer innerhalb der ersten sechs Wochen nach der Entbindung auftretenden Psychose unabhängig von Psychopathologie und Ätiologie. Manche Autoren grenzen hiervon die „Laktationspsychose“ ab, die in der Zeit von sechs Wochen nach der Geburt bis zum Ende des ersten Jahres gerechnet wird. Letztere Unterscheidung wird bei den Begriffen „postpartale

Postpartale Psychosen imponieren durch heterogene und atypische psychopathologische Bilder. Die nosologische Zuordnung zu einer in sich geschlossenen Krankheitsentität misslingt. Statt dessen können - geordnet nach Häufigkeit - affektive, schizoaffektive, schizophreniforme, schizophrene und organische postpartale Psychosen beobachtet werden, mit einer Gesamtprävalenzrate von ein bis zwei Promille. Sie stellen damit die seltensten postpartalen psychiatrischen Störungen dar. Auch ihre Ätiologie ist heterogen: neben hormonell-neurobiologischen Faktoren sind epidemiologisch-soziale und gynäkologische Einflüsse von Bedeutung. Gemeinsames Kernsymptom aller postpartaler Psychosemanifestationen ist die Beziehungsstörung zum Kind, die zu seltenen, dann aber oft schwerwiegenden Gefährdungen des Kindes bis zum erweiterten Suizid und Infantizid führen kann. Demzufolge ist eine frühzeitige Diagnosestellung und eine stationäre am psychopathologischen Prägnanztyp orientierte Behandlung - nach Möglichkeit in einer Mutter-Kind Einheit - erforderlich, bei der die Versorgungs- und Erziehungsfähigkeit nach Abklingen der postpartalen psychotischen Symptomatik festgestellt werden kann. Insgesamt ist der Verlauf postpartaler Psychosen eher positiv, insbesondere dann, wenn medikamentöse rezidivprophylaktische Maßnahmen ergriffen werden und eine kontinuierliche ambulante Weiterbehandlung etabliert werden kann. Bei anamnestisch abgelaufener postpartaler Psychose kann sogar nach erneuter Entbindung an eine prophylaktische medikamentöse Intervention gedacht werden; hier sollte jedoch eine Abwägung gegenüber dem dann manchmal erforderlichen Abstillen vorgenommen werden.

Psychose“ oder „Postpartumpsychose“ aufgegeben. Der Begriff „peripartale Psychose“ erweitert den zeitlichen Bezug zum Geburtstermin noch stärker als die vorhergenannten Begriffe. Daneben ist auch der Begriff „postnatale Psychose“ in Gebrauch, hier wird die Perspektive von der Mutter (partus = Geburt) zur Perspektive aus Sicht des Kindes (neonatus $=$ Neugeborenes) verschoben . In der offiziellen ICD-10-GM-Version von 2005 wird unter der Ziffer F 53.1 eine „schwere psychische und Verhaltensstörung im Wochenbett, andernorts nicht klassifiziert" aufgeführt und als Beispiel die „Puerperalpsychose ohne nähere Angaben“ genannt. In der DSM-IV-Klassifikation fehlt eine eigene Rubrik für postpar- tale Psychosen. Statt dessen kann für die kurze psychotische Störung (DSM-IV: 298.8) bzw. für folgende affektive Störungen: Depressive, manische oder gemischte Episode bei, Bipolar 1 oder Bipolar 2-Störung, sowie der majoren Depression (DSMIV: 296) eine Zusatzkodierung „mit postpartalem Beginn“ vorgenommen werden, wenn die genannten Störungen innerhalb von vier Wochen nach einer Entbindung aufgetreten sind. Darüber hinaus werden Psychosen, die sich postpartal zum ersten Mal manifestieren und vorbestehende Psychosen, die im Wochenbett erneut exazerbieren, in der Literatur unter postpartaler Psychose subsumiert. Wir wollen im Folgenden versuchen, durch Darstellung 
der Epidemiologie, der Psychopathologie, der Ätiologie, des Verlaufs und der Therapie zu vermitteln, welche (heterogenen) Krankheitsbilder sich hinter dem Begriff der „postpartalen Psychosen“ verbergen.

\section{Zur Epidemiologie post- partaler Psychosen}

Die Geburt eines Kindes ist für viele Frauen eines der eindrucksvollsten Erlebnisse. Das Wochenbett stellt im Gegensatz zur Schwangerschaft eine Zeit erhöhter Vulnerabilität für psychiatrische Störungen dar; die psychischen Anforderungen der Mutterschaft (und in geringerem Umfang auch der Vaterschaft) sind beim Menschen relativ störbarer als bei anderen Säugern, möglicherweise weil sie weniger instinktgebunden sind. Ca. 25-50\% der Frauen erleben in den ersten zwei bis fünf Tagen nach der Entbindung den sogenannten „Baby-Blues“, eine kurzdauernde affektive Störung mit affektiver Labilität, teilweise depressivem Herabgestimmtsein, Gereiztheit und auch manchmal mit subeuphorischen Auslenkungen. In der Majorität der Fälle klingt diese Symptomatik ohne Behandlung ab. Ausgeprägtere Formen können in postpartale depressive Störungen übergehen, die jedoch keinen psychotischen Charakter haben. Die Prävalenz dieser Störung soll bei ca. 10-15\% liegen (20, 34). Allerdings sind in einer aktuellen Untersuchung mit einer auf einem strukturierten klinischen Interview beruhenden Diagnosestellung, die gegenüber einem Fragebogen-gestützten Design von einer klinisch orientierten, engeren Depressionsdefinition ausgeht, niedrigere Prävalenzraten - zumindest im Süddeutschen Raum - erhoben worden (31). Die postpartalen Psychosen mit einer Prävalenz von ein bis zwei von tausend Frauen $(0,1-0,2 \%)$ stellen die seltensten psychiatrischen Auffälligkeiten im Wochenbett dar $(20,34)$. Laut Statistischem Bundesamt (2004) wurden im Jahre 2003 etwa 715290 Kinder geboren. Bei einer Prävalenzrate von ein bis zwei Promille würde dies bedeuten, dass die zu erwartende Anzahl postpartaler Psychosen bundesweit zwischen ca.
715 und 1430 erkrankter Frauen läge. Laut Krankenhausstatistik des statistischen Bundesamtes gab es im Jahr 2000 allerdings nur 251 mit einer F53-Diagnose aus dem Krankenhaus entlassene vollstationäre Patientinnen.

\section{Psychopathologie und nosologische Zuordnung}

In der Vergangenheit waren akute hirnorganische klinische Bilder wie Fieberdelirien etc. („Kindbettfieber“) recht häufig zu beobachten. Zumindest in den westlichen Industrienationen spielen diese organischen postpartalen Psychosen seit Einführung der Antibiotikabehandlung von Wochenbett-Infektionen praktisch nur noch eine untergeordnete Rolle. Hirnorganisch getönte postpartale Psychosen können jedoch nach starken Blutverlusten unter der Geburt ab und zu auftreten. Bei einem Vergleich psychopathologischer Variablen von 58 postpartalen Psychosen mit 52 Psychosen von jungen Frauen, die ohne Zusammenhang zum Wochenbett standen, stellte sich in etwa einem Viertel der psychopathologischen Variablen Unterschiede heraus: Postpartal erkrankte Frauen wiesen mehr manische Symptome, oneiroides Erleben und Verworrenheit auf, während die Gruppe, deren Psychoseausbruch nicht in Zusammenhang mit der Entbindung stand, mehr schizophrene Symptome zeigten. Bezogen auf die Research-Diagnostic-Criteria (RDC) zeigte sich, dass nur 5 der 58 postpartalen Psychosen die RDC-Kriterien von Schizophrenie erfüllten. Die meisten postpartalen Psychosen wurden als schizomanische oder schizodepressive Episoden klassifiziert (5). Darüber hinaus wurde versucht, postpartale Psychosen nach der Leonhardschen nosologischen Zuordnung zu beschreiben. Hierbei war ein hoher Anteil (19 von 42 postpartalen Psychosen) als zykloide Psychosen identifizierbar, neben vier weiteren psychopathologischen Prägnanztypen. Somit bestätigt sich innerhalb des differenzierten Leonhardschen Klassifikationssystems ebenfalls die Heterogenität postpartaler Psychosen (22, 23). Häufige Initialsymptome postparta- ler Psychosen sind Angst, Unruhe, depressive Verstimmung, Schlafstörungen, Verhaltensauffälligkeiten, katatone Erregungszustände, Wahnvorstellungen und Halluzinationen (36). Von 119 Frauen, die an einer schweren postpartalen Psychose litten, zeigten 57\% Symptome einer affektiven Psychose, 18\% Symptome einer schizoaffektiven Psychose, 12\% Symptome einer schizophreniformen Psychose, 4\% Symptome einer kurzen reaktiven Psychose und 9\% schizophrene Symptome. Von den Patientinnen mit Erkrankungen aus dem schizophrenen Spektrum zeigten viele die Symptomatik zykloider Psychosen sowie Zeichen von Verworrenheit. Patienten mit frühem Beginn der Symptomatik (innerhalb von zwei Wochen nach Entbindung) zeigten häufiger manische Symptome, Symptome von zykloiden Psychosen, oneiroide Zustände und Verworrenheitsanzeichen (39-42). Der Eindruck, dass postpartale Psychosen keine nosologische Entität darstellen, wird unterstützt durch eine Studie an 636 Frauen, die während ihrer Vorstellung in einer NotfallAmbulanz psychiatrisch untersucht worden sind: 15 von diesen litten an postpartalen Psychosen, 11 an affektiven Psychosen sowie vier an schizophreniformen Psychosen. Bei fünf Patientinnen manifestierten sich katatone Symptome (21). Neben der psychopathologischen und nosologischen Heterogenität und einer im Vergleich zu den klarer definierten non-postpartalen Psychosependants zu beobachtenden Atypizität der Psychopathologie (oneiroide und hirnorganisch getönte Querschnittssymptomatik) besteht jedoch im Kern eine grundlegende Gemeinsamkeit, die bei fast allen postpartal psychotisch erkrankten Frauen zu beobachten ist: Es handelt sich um eine schwere Beziehungsstörung dem Kind gegenüber und daraus entstehende Schuldgefühle, da die betroffenen Frauen nicht ihrer eigenen Erwartung an sich selbst als Mutter entsprechen. Dieses Kernsymptom kann negative Effekte auf die Qualität der Mutter-Kind-Beziehung, das Bindungsverhalten sowie auf die kognitive und emotionale Entwicklung des Kindes haben (15, 
28). In seltenen Fällen kann die Beziehungsstörung zum Kind so ausgeprägt sein, dass z.B. bei Vorliegen eines depressiv bedingten nihilistischen Wahns dieser auf das Kind übertragen wird und es zu einem erweiterten Suizid kommt. Auch bei schizophreniformen oder schizophrenen postpartalen Psychosen der Mütter können sich - ebenfalls selten (1:50 000) - Infantizide ereignen, insbesondere dann, wenn das Kind im Zentrum des Wahnerlebens steht $(6,27,46)$. Um diesem Risiko zu begegnen, ist eine Früherkennung postpartaler Psychosen wichtig. Darüber hinaus bedarf es spezieller therapeutischer Angebote z.B. in Mutter-Kind Behandlungseinheiten, die die Versorgungs- und Erziehungsfähigkeit erkrankter Mütter fördern (siehe auch: „Therapie“). Die forensisch-psychiatrische Beurteilung eines von der Mutter überlebten erweiterten Suizidversuchs oder eines Infantizids richtet sich nach den Vorschriften der Paragraphen 20 und 21 des Strafgesetzbuches (Schuldunfähigkeit, erheblich verminderte Schuldfähigkeit).

\section{Auslöser und Ätiologie}

Aus den Ergebnissen, dass eine gleichbleibende Inzidenzrate für postpartale Psychosen unabhängig von kulturellen und ethnischen Besonderheiten gefunden wurde, ist auf eine endogene Ätiologie dieser Psychosen geschlossen worden, die durch die physiologischen Umstände der Geburt getriggert werden (19). So wird eine Sensitivitätserhöhung im dopaminergen System durch postpartalen „Östrogen-Entzug“ diskutiert (49). Auslösende Faktoren können hierbei in der Länge des Geburtsverlaufs sowie in einer Geburt während der Nachtstunden gesehen werden (44). Darüber hinaus werden der Erstgebärendenstatus, genetische Prädisposition und die abrupten hormonellen Veränderungen als Auslöser genannt (42). In Einzelfallbeschreibungen wird die zeitliche Nähe zwischen postpartaler Psychose und postpartaler Thyreoiditis herausgestellt (4). Darüber hinaus kann eine postpartale Psychose verstärkt oder sogar ausgelöst werden durch höhere Do-

\section{Tab. 1 Vorteile der gemeinsamen Behandlung von postpartal psychotisch erkrankten Müttern und ihren Kindern in einer Mutter-Kind Einrichtung}

- Trennungszeiten von Mutter und Kind werden reduziert

- Förderung der mütterlichen Identität durch weitgehenden Verbleib in der Mutterrolle

- Kinderversorgung während der individuellen Therapiezeiten der Mutter oder bei etwaiger Dekompensation der Mutter durch Fachpersonal sichergestellt

- Bindung des Kindes an die Mutter als wichtigste Bezugsperson bleibt weitestgehend erhalten

- Mutter-Kind Interaktion als zentraler Gegenstand von Diagnostik und Therapie unter Zuhilfenahme von audiovisuellen Verfahren (gezieltes Video-Feedback)

- Erweiterung von Diagnostik und Therapie auf Mutter-Vater-Kind Interaktion

- Unterstützung der Mutter im Erlernen oder Aufrechterhalten der alltäglichen Versorgung des Kindes

- Valide Beurteilung der Versorgungs- und Erziehungsfähigkeit der Mutter sowie des regulativen Potentials des Mutter-Kind Systems

- Gute Einschätzungsmöglichkeit der familiären, väterlichen und sonstigen sozialen Unterstützungsressourcen

- Bearbeitung vorübergehender oder endgültiger Trennung der Mutter vom Kind

sen von Bromocriptin, einem $\mathrm{D}_{2}$-Rezeptor-Agonist, der u.a. zum Abstillen eingesetzt wird (33). Ein erhöhtes Risiko, postpartale Psychosen zu entwickeln, haben insbesondere Frauen mit einer positiven Familienanamnese für psychiatrische Störungen sowie bei eigenen psychiatrischen Vorerkrankungen (34). $\mathrm{Zu}$ folgenden Faktoren wurde in einer prospektiven Studie kein $\mathrm{Zu}$ sammenhang zu einem erhöhten Risiko, postpartale Psychosen zu entwickeln, festgestellt: materielle Probleme, zwischenmenschliche Schwierigkeiten, geringe Unterstützung während der Schwangerschaft, negative Einstellung der Frau zur Schwangerschaft oder gesundheitliche Einschränkungen durch die Schwangerschaft (25).

\section{- Verlauf}

Frauen mit einer bestehenden Psychoseerkrankung haben ein hohes Risiko im Wochenbett eine erneute Episode dieser Erkrankung zu erfahren. Insbesondere bei vorbestehenden affektiven Psychosen ist die Wahrscheinlichkeit, nach einer Entbindung eine erneute affektpsychotische Episode zu entwickeln gegenüber Frauen, die nicht entbunden haben, etwa verdoppelt (17). Darüber hinaus ist eine deutliche Risikoerhöhung für postpartale Psychosen gegeben, wenn nach einmaliger postpartaler Psychose erneute Schwangerschaften erfolgen. Bei
Frauen mit einer postpartalen Erstmanifestation einer psychotischen Episode ist in der Folge von einem erhöhten Risiko psychotischer Episoden unabhängig von Schwangerschaft und Wochenbett auszugehen (35). Generell verlaufen jedoch Psychosen, die ausschließlich postpartalen Charakter haben, günstiger und mit weniger Residualsymptomatik als Psychosen, deren Auslösung und Rezidiv nicht an das Wochenbett gebunden sind $(2,32)$. Neben dem Wochenbett wird das Klimakterium als Zeitraum hervorgehoben, in welchem häufig Rückfälle ehemaliger postpartaler Psychosen zu erwarten sind $(37,38)$, manchmal verschlechtert sich der psychopathologische Zustand zyklusabhängig im Rahmen der bekannten prämenstruellen hormonellen Schwankungen. Insgesamt ist herauszustellen, dass der Verlauf der psychopathologisch heterogenen postpartaler Psychosen - möglicherweise auch aufgrund der vorherrschenden affektiv psychotischen sowie schizoaffektiven Bilder - als besser einzuschätzen ist als bei schizophrenen Psychosen (2). Im Allgemeinen soll eine Frühmanifestation postpartaler Psychosen innerhalb der ersten 14 Tage nach Entbindung stärker mit einem affektpsychotischen Verlauf korrelieren, während eine spätere Manifestation zu einem stärker schizophreniform geprägten Verlauf disponieren soll. 


\section{Therapie}

Im Grundsatz ist die psychopharmakologische Behandlung bestehender akuter postpartaler Psychosen - je nach syndromatischer Zuordnung - ähnlich den vergleichbaren psychotischen Episoden ohne Zusammenhang zum Wochenbett (45). Je nach eingesetztem Medikament und bei entsprechenden Dosierungen ist Abstillen in Erwägung zu ziehen. Aufgrund der dargestellten Kenntnisse über den Verlauf postpartaler Psychosen und der hohen Rezidivwahrscheinlichkeit bei erneuter Entbindung kann es sinnvoll sein, im Wochenbett eine prophylaktische Behandlung der Mutter zu beginnen, auch wenn noch keine Symptome eines Psychoserezidivs zu erkennen sind, demgegenüber die individuelle Rückfallwahrscheinlichkeit bei der betroffenen Frau jedoch sehr hoch erscheint, um einer Trennung von Mutter und Kind vorzubeugen. Stellt sich gegen die Erwartung kein Rückfall ein, kann die Medikation beginnend etwa in der sechsten Woche postpartum vorsichtig reduziert werden. Dies sollte in gynäkologischfachärztlicher Zusammenarbeit erfolgen. Da die meisten postpartalen Psychosen schwerwiegende psychiatrische Erkrankungen darstellen, wird in vielen Fällen eine stationäre Behandlung erforderlich sein. Bei psychotischen Depressionen kann auch der Einsatz von Schlafentzug eine effektive Ergänzung der psychopharmakologischen Therapie sein (47). In Großbritannien und den USA wird bei endogen wirkenden depressiven postpartalen Psychosen nicht selten auch Elektrokrampftherapie vorgeschlagen. $\mathrm{Ob}$ sich in der Behandlung postpartaler Psychosen Behandlungen mit Östrogenen oder anderen Hormonen durchsetzen werden oder nicht bleibt abzuwarten $(1,3)$. Bei stärkerer affektpsychotischer Betonung im Verlauf sollte der Einsatz von Mood-Stabilizern wie Lithium, Carbamazepin oder Valproinsäure in Erwägung gezogen werden. Sobald die psychische Stabilität der Mutter es erlaubt, ist auf eine Behandlung in Mutter-Kind-Einheiten hinzuwirken $(11,12,13)$, eine Behandlungs- form, die in England und Australien sowie inzwischen in Frankreich nahezu als Standardbehandlung postpartaler psychotisch erkrankter Frauen etabliert ist. Diese hat den Vorteil, dass der Kontakt zum Kind so schnell wie möglich wiederhergestellt wird oder - bei günstigem Verlauf - von Anfang an beibehalten wird und Gegenstand der therapeutischen Bemühungen wird $(7,16,26$, 30,48 ). Wichtig in diesem Behandlungssetting ist allerdings, dass ausreichend erfahrene Pflegepersonen, am besten einige Kinderkrankenschwestern im Behandlungsteam sind, die eine separate Versorgung des Kindes dann übernehmen können, wenn die Mutter krankheitsoder therapiebedingt trotz Anleitung und Unterstützung nicht dazu in der Lage ist (siehe Tab. 1). Neben den allgemeinen Aspekten der Psychosen-Psychotherapie (14) wird zum Teil durch audiovisuelle Verfahren unterstützt - die Interaktion zwischen Mutter und Kind bearbeitet. Im geschützten Rahmen einer stationären Mutter-Kind Einrichtung kann die Versorgungs- und Erziehungsfähigkeit der Mutter sowie die supportive Funktion der Familie und des sozialen Umfelds mit hoher Validität eingeschätzt werden. Hierbei sind die männlichen Partner postpartal psychotisch erkrankter Frauen mit einzubeziehen $(8,9,10)$. In vielen Fällen - selbst bei anfangs ausgeprägten postpartalen Psychosen - wird mit diesem Konzept ein guter Therapieerfolg erreicht und die Hauptverantwortung für das Kind kann bei der Mutter verbleiben. Manchmal ist jedoch das Jugendamt einzubeziehen und als ultima ratio eine Unterbringung des Kindes z.B. in eine Pflegefamilie unumgänglich, wenn trotz aller Bemühungen eine ausreichende Rekompensation der Mutter nicht gelingt.

\section{Postpartum psychoses}

Postpartum psychoses show heterogeneous and atypical psychopathology. They cannot be easily classified as one nosological entity: according to their frequency the most common manifestations of postpartum psychoses are affective psychotic disorders, schizoaffective disorders, schi- zophreniform psychotic disorders, schizophrenia and organic psychotic disorders. The prevalence rate of all types of postpartum psychoses is 1 to 2 out of 1000 . Concerning the aetiology and the precipitating factors of postpartum psychoses numerous variables are discussed: changing hormone levels, and neurobiological, epidemiological, social, and gynaecological factors as well. An important and the most common symptom of all types of postpartum psychoses is the disturbed mother-child relationship. Although rare phenomena, maternal aggression, infanticidal thoughts, and infanticidal behaviour represent severe manifestations of the disturbed mother-child relationship. Early detection and consequent therapy of this possibly dangerous behaviour is essential. Inpatient treatment is required and has to be established as soon as possible. If the inpatient treatment takes place on a motherchild unit subsequent to remission of the psychotic symptoms, assessment of the mother's parenting abilities is also necessary. In general the course and outcome of postpartum psychoses is positive. Maintaining neuroleptic medication or prescription of mood stabilizers can reduce the risk of relapse; consequent outpatient treatment is required. After the next parturition the recommendation of a prophylactic medication may be helpful if a known history of postpartum psychoses exists. In these cases $a b$ lactation has to be considered according to the type and dosage of the medication taken.

\section{Key words}

postpartum psychosis - postnatal psychosis - puerperal psychosis mother-child unit - treatment

\section{Literatur bei der Redaktion oder unter www.psychoneuro.info}

